\title{
Hyperpolarizability of Water at the Air-Vapor Interface: Numerical Modeling Questions Standard Experimental Approximations
}

\author{
Guillaume Le Breton, Oriane Bonhomme, Pierre-François Brevet, Emmanuel \\ Benichou, and Claire Loison* \\ University of Lyon, Université Claude Bernard Lyon 1, CNRS, Institut Lumière Matière, \\ F-69622, Villeurbanne, France \\ E-mail: claire.loison@univ-lyon1.fr
}




\begin{abstract}
We investigate the molecular first hyperpolarizability of water molecules nearby the liquid-vapor interface. The hyperpolarizability of each molecule is calculated at the quantum level within an explicit, inhomogeneous electrostatic embedding. We report that the average molecular first hyperpolarizability tensor depends on the distance relative to the interface, but it practically respects the Kleinman symmetry everywhere in the liquid. Within this numerical approach, based on the dipolar approximation, the water layer contributing to the Surface Second Harmonic Generation (S-SHG) intensity is less than a nanometer. We show that within this interfacial layer, the common assumption considering a single, constant hyperpolarizability for all water molecules is not supported by our data: hyperpolarizability fluctuations are expected to impact the S-SHG intensity. These results represent a step forward the molecular interpretation of experimental S-SHG signal of aqueous interfaces.
\end{abstract}

\title{
Graphical TOC Entry
}

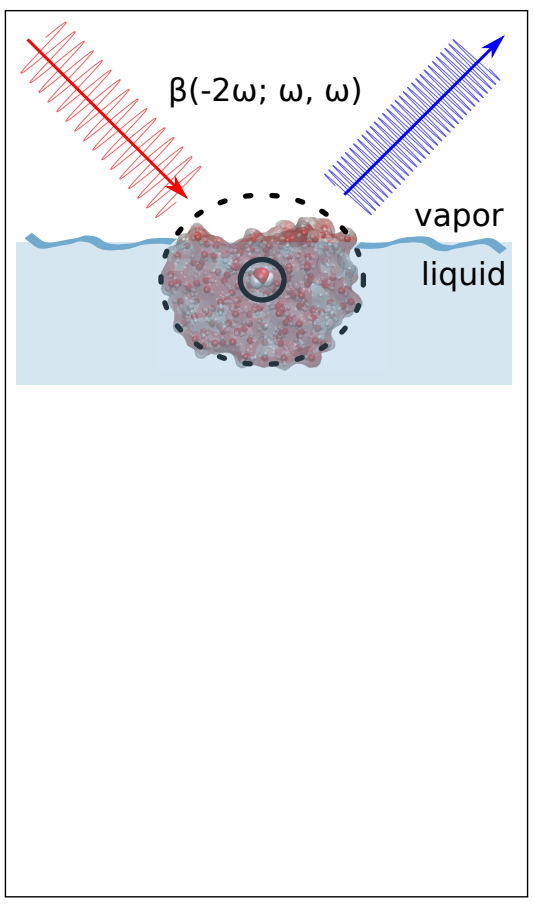




\section{Keywords}

Water, Interface, Second Harmonic Generation, Molecular Dynamics, Quantum Chemistry, Hyperpolarizability 
Liquid water is ubiquitous on Earth; understanding the properties of its interfaces with other materials is essential for many fields of Physics, Chemistry, and Biology. Surface analytical tools have been developed to obtain information on the molecular organization at interfaces ; in particular, non-linear optical techniques such as Surface Second Harmonic Generation (S-SHG) and Surface Sum Frequency Generation (S-SFG) are increasingly used to probe the structure of liquid-gas ${ }^{1-8}$, liquid-liquid ${ }^{9,10}$ or liquid-solid ${ }^{11-15}$ interfaces. The SHG is an optical process whereby two photons at a fundamental frequency are converted into one photon at twice the fundamental frequency, i.e. the harmonic frequency. Coherent SHG techniques are surface-sensitive because of the cancellation of the SHG process within centrosymmetric media like bulk liquids. ${ }^{16}$ Moreover, the SHG response is strongly dependent on the molecular arrangement and the molecular electrostatic environments, so that S-SHG has become a useful tool for probing molecular structure ${ }^{1,17,18}$ or surface potential. ${ }^{7,8,11,13,19,20}$ However, linking the experimental S-SHG intensity to interface molecular structure often remains a challenge, for which different experimental characterizations, ${ }^{8,10}$ theoretical developments, ${ }^{16,21}$ and molecular modeling ${ }^{22-24}$ can benefit from each others. ${ }^{9,25-28}$

The interpretation of the S-SHG signal of the water/air interface has been a subject of debate for decades. ${ }^{8,9,29}$ Efforts have been made to assess the contribution of the interface (local) contribution of "the Bonded Interface Layer" (BIL) and the one of bulk electric quadrupole and magnetic dipole contributions to the SHG signal appearing in the diffuse layer (DL). ${ }^{8,23}$

For a neat water/air interface, Dalstein et al. ${ }^{8}$ and Zhang et al. ${ }^{29}$ attribute the signal to the BIL, i.e. the layer where the inversion symmetry is broken by the interface-specific structure. Typically, under the electric dipole approximation, this contribution to the quadratic surface susceptibility tensor $\chi^{(2)}$ is modeled as a sum of the molecular hyperpolarizability tensors $\beta_{i j k}^{n}\left(i, j, k\right.$ are directions of the molecular frame $\{a, b, c\}$ of the $n$-th molecule). ${ }^{9,29}$ To express $\chi^{(2)}$ in the laboratory frame, one takes into account the orientation of the $n^{\text {th }}$ molecule via the matrix $\mathbf{R}^{n}$ of the $n^{\text {th }}$ molecular coordinate system $\{a, b, c\}$ in the laboratory 
frame $\{X, Y, Z\}: \chi^{(2)} \propto \sum_{n} \mathbf{R}^{n} \mathbf{R}^{n} \mathbf{R}^{n} \beta^{n}{ }^{9,29}$ To interpret the S-SHG signal of the water/air interface in terms of interface structure using this formalism, one therefore needs to know the hyperpolarizabilities of individual water molecules. ${ }^{29}$

On the one side, the molecular hyperpolarizability is commonly assumed to be the same for all the water molecules of a given phase, and respecting the molecular symmetry group $C_{2 v}$. These hypotheses permit to extract from the susceptibilities the mean molecular orientation - via the orientation matrices. ${ }^{1,2}$ On the other side, it is known that solvation has a substantial effect on the measured hyperpolarizability of water molecules, and the liquid environment breaks the $C_{2 v}$ symmetry. ${ }^{30-35}$ To reach quantitative interpretation of S-SHG signal of aqueous interfaces, there is an urgent need to determine the water hyperpolarizability values, precisely at these interfaces.

In particular, the origin of the failure of Kleinman symmetry observed experimentally for the neat air/water interface $\left(\chi_{Z X X}^{(2)} \neq \chi_{X Z X}^{(2)}\right.$, for example) is debated. ${ }^{29,36,37}$ It was attributed by Zhang et al. to the asymmetry of the molecular hyperpolarizability, for instance to $\beta_{a c a} \neq \beta_{c a a}$ or $\beta_{b c b} \neq \beta_{c b b}$, where the $c$-axis is along the water dipole moment. ${ }^{29}$ Such a symmetry breaking indeed exists for water in the gas phase, ${ }^{32}$ but to our knowledge, it has been reported neither in the bulk liquid, nor at the interface.

In this Letter, we report theoretical chemistry calculations of the molecular hyperpolarizability of water molecules nearby the interface, and discuss the results in terms of the S-SHG measurements. Liquid water is a difficult system for computational modeling in general, and for the prediction of electric properties in particular because of the extended H-bond network. ${ }^{38,39}$ Modeling the molecular environment as homogeneous dielectric material is not sufficient to reproduce the sign change of water hyperpolarizability from the gas phase to the liquid phase. ${ }^{40}$ Here, we propose a sequential Quantum Mechanical/Molecular Mechanics (QM/MM) approach to provide new insights on the contribution of water molecular hyperpolarizability to the S-SHG signal of the liquid-vapor interface, see Figure 1. Such 
(a) MD

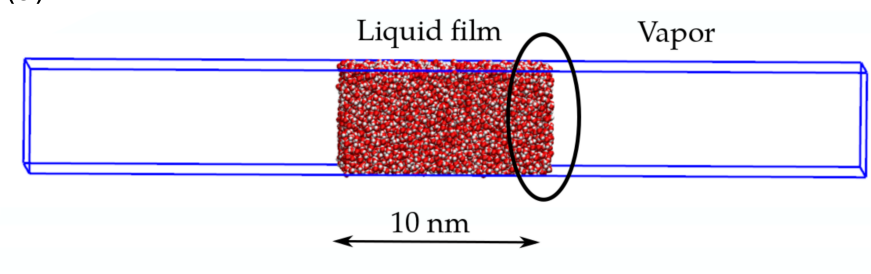

(b) Interface

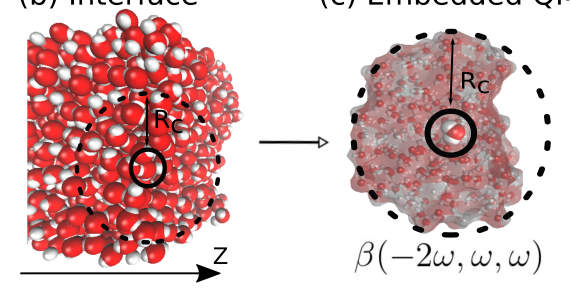

Figure 1: Scheme of the numerical procedure performed by the home-made software FROG. (a) Molecular Dynamics snapshot of the water liquid-vapor simulation within a box of $5 \times 5 \times 40 \mathrm{~nm}^{3}$. (b) Zoom on the interface at a given time step. Average property profiles are obtained as a function of the coordinate normal to the interface $(Z)$ by selecting and analyzing molecules within $0.1 \mathrm{~nm}$-thick slices. (c) A single water molecule with its electrostatic environment, within a radius $R_{c}$, used for Quantum Mechanics calculation of individual hyperpolarizabilities.

methods have been successful to describe the impact of solvation on the optical properties of NLO-dyes, ${ }^{24,41-46}$ and on other non-resonant molecules. ${ }^{31-33,35,47-49}$ First, a classical molecular description of the structure of the liquid-vapor water interface is obtained using Molecular Dynamics (MD) simulations. Then, the frequency dependent $\beta(-2 \omega, \omega, \omega)$ of each water molecule at the interface is computed using a QM description within an electrostatic embedding framework ${ }^{50}$ to mimic the condensed phase environment.

In the following, we briefly present the MD and QM steps, before discussing the evolution of the hyperpolarizability when the water molecule gets close to the liquid-vapor interface.

The structure of the liquid-vapor interface has been obtained by an MD simulation, using LAMMPS. ${ }^{51}$ Figure $1(\mathbf{a})$ shows the simulation box. Using 3D periodic boundary conditions in a $5 \times 5 \times 40 \mathrm{~nm}^{3}$ box, a water film surrounded by vapor is simulated at $300 \mathrm{~K}$. The two liquid-vapor interfaces are parallel to the $\{X, Y\}$ plane, but we shall focus on a single interface here. Thanks to the sufficient film thickness (about $10 \mathrm{~nm}$ ), the middle part of the fluid film well mimics bulk water.

We shall focus first on the density profile and the water dipole orientation profile displayed in Figure 2 (b). The density decreases progressively from the bulk value to zero with a profile 


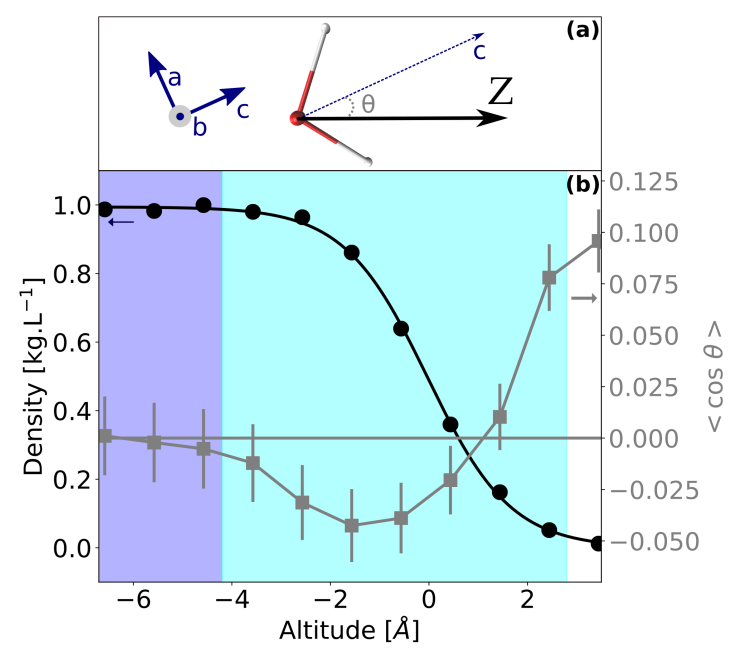

Figure 2: (a) Definition of the molecular frame $\{a, b, c\}$ and of the angle $\theta=(c, Z)$ characterizing the orientation of the water dipole moment with respect to the interface normal $Z$-axis. (b) Water density profile (black circles) and $\langle\cos \theta\rangle$ profile (gray squares) as a function of the altitude. The solid black line is proportional to a hyperbolic tangent function fitted onto the density profile. The error bars are calculated using Eq. S3.

close to a hyperbolic tangent shape. ${ }^{52}$ Its inflection point permits to define an absolute position $Z_{0}$ for the interface, and the distance relative to it $\left(\Delta Z=Z-Z_{0}\right)$ that is called "altitude" here.

The projection of the water dipole $c$-axis on the interface normal $Z$ is particularly relevant for the interpretation of the S-SHG intensities, see Figure 2 (a). Indeed, the $C_{\infty}$ symmetry of the interface, associated to an expected $C_{2 v}$ symmetry of the water hyperpolarizability, yields surface susceptibility components expressed as linear combinations of $\left\langle\beta_{i j k}(\cos \theta)^{l}\right\rangle$ where $\theta$ is the $(c, Z)$ angle ${ }^{29,53}$ and $l$ is 1,2 or 3 - see Section S4 for complete expressions. In particular, we focus here on the $\chi_{Z Z Z}^{(2)}$ element since it is the dominant one, according to experimental observations on the water-air interface: ${ }^{9}$

$$
\chi_{Z Z Z}^{(2)} \propto\left\langle\beta_{\perp} \cos \theta\right\rangle+\left\langle\left(\beta_{c c c}-\beta_{\perp}\right) \cos ^{3} \theta\right\rangle
$$


where $\langle\cdot\rangle$ defines the average over molecules and

$$
\beta_{\perp}=\left(\beta_{c a a}+\beta_{c b b}+2 \beta_{a c a}+2 \beta_{b c b}\right) / 2 .
$$

The profile of $\langle\cos \theta\rangle$ is therefore plotted in Figure 2 (b) as a function of the altitude. The region where a net orientation appears, i.e. $\langle\cos \theta\rangle \neq 0$, spotlights the BIL and the thickness of the layer which should contribute to the S-SHG signal. Noticeably, the net water orientation appears few Angstroms further away from the interface than the density drop. The deviation for the H-bond network relative to the bulk occurs at a similar altitude (see Figure S3).

Moreover, $\langle\cos \theta\rangle$ provides structural information: negative values indicate that the dipole moment of the water molecule points on average with the hydrogens towards the bulk phase. But this propensity is weak, and the dipole moment of the water molecule in the most external water layers is preferentially close to parallel to the interface plane (see Figure S4). These results agree with previous studies. ${ }^{9,39,54}$

To guide the interpretation, we have divided the system into 3 regions shown in Figure 2: bulk (in purple), interface (in cyan) and vapor (in white). The limit between the bulk and interface is fixed at the altitude where $\langle\cos \theta\rangle$ significantly deviates from its bulk value. The edge of the vapor area is defined where the density reaches $5 \%$ of the bulk value. In practice, we have set the vapor region at the point where accurate statistical averaging over the number of water molecules became difficult because of too small densities.

The second step consists in computing, for each MD snapshot, the molecular hyperpolarizability $\beta(-2 \omega, \omega, \omega)$ of all water molecules. For the QM calculation, we use the Polarizable Embedding $(\mathrm{PE})$ scheme at the zeroth order ${ }^{50,55}$ as implemented in the software DALTON ${ }^{56}$ An environment up to a cutoff distance $R_{c}$ is built from the MD trajectory, see Figure 1 (b) (c). Each surrounding molecule is described as a point-charge and affects 
the Hamiltonian of the target molecule through an electrostatic potential, see Section S1.1. The same electrostatic description of the water molecule is used for the MD and the PE embbeding for consistency. In the QM calculation, the total inhomogeneous electrostatic field induced by all surrounding molecules is taken into account. Using this single-molecule electrostatic embedded Hamiltonian, the electronic states of each molecule are approached at a Density Functional Theory (DFT) level. Then, the optical properties such as hyperpolarizability tensor elements $\beta(-2 \omega, \omega, \omega)$ are computed using a frequency-dependent quadratic response scheme. ${ }^{57}$

This procedure has been widely used for homogeneous phases, ${ }^{31,33,38,58,59}$ or biomolecules,${ }^{43}$ but much less at phase separations. ${ }^{42}$ These methodological choices were validated by comparing our results to the ones obtained with more accurate schemes for water molecules in vacuum and bulk liquid phases, ${ }^{32,35}$ see Tables $1, \mathrm{~S} 1$ and S2.

Table 1: Molecular SHG hyperpolarizability $\beta(-2 \omega, \omega, \omega)$ in the gas and bulk liquid water phases. Hyperpolarizabilities are in a.u. The excitation wavelength $\lambda$ is in nm. For the liquid phase, the mean values are presented along with the distribution withdith in brackets. Table S1 reports the components that average to 0 .

\begin{tabular}{lcccccc}
\hline & \multicolumn{2}{c}{ Gas } & & \multicolumn{3}{c}{ Liquid } \\
\cline { 2 - 3 } \cline { 5 - 6 }$\lambda$ & $\infty^{a}$ & $800^{a}$ & & $\infty^{b}$ & $\infty^{a}$ & $800^{a}$ \\
\hline$\beta_{c a a}$ & -10.6 & -12.5 & & $-2.1[1.1]$ & $-1.9[1.0]$ & $-1.9[1.1]$ \\
$\beta_{a c a}$ & -10.6 & -12.4 & & $-2.1[1.1]$ & $-1.9[1.0]$ & $-2.0[1.1]$ \\
$\beta_{c b b}$ & -4.2 & -5.0 & & $1.6[1.8]$ & $2.6[1.7]$ & $2.7[2.0]$ \\
$\beta_{b c b}$ & -4.2 & -7.4 & & $1.6[1.8]$ & $2.6[1.7]$ & $2.3[2.1]$ \\
$\beta_{c c c}$ & -12.1 & -15.3 & & $3.0[3.0]$ & $3.9[2.8]$ & $4.0[3.1]$ \\
\hline
\end{tabular}

${ }^{a}$ : this work, using DFT-CAM-B3LYP/d-aug-pVTZ level within the PE-0 response scheme using the charges of the TIP4P-2005 force field. The electrostatic environment is built up to $R_{c}=2 \mathrm{~nm}$ around the target molecule. ${ }^{b}$ : Combined coupled cluster/molecular mechanics (CC/MM) method by Liang et al. ${ }^{35}$

Once the methodology validated for a water molecule in the gas phase and in bulk liquid water, the hyperpolarizabilities were calculated at the interface with the same approach. In 
the interface region, as in the bulk region, the autocorrelation time of the hyperpolarizability of a single water molecule nearby the interface is of the order of a few picoseconds (see Figure S6). Therefore, we exploited the MD configurations obtained every 20 ps of simulation. Then, we have investigated the impact of the environment sampling: approximately 4000 configurations are needed to converge the average hyperpolarizabilities at a given altitude (see the Method section, Figures S7 and S8). MD trajectories about 2 ns are therefore necessary in our QM/MM scheme.

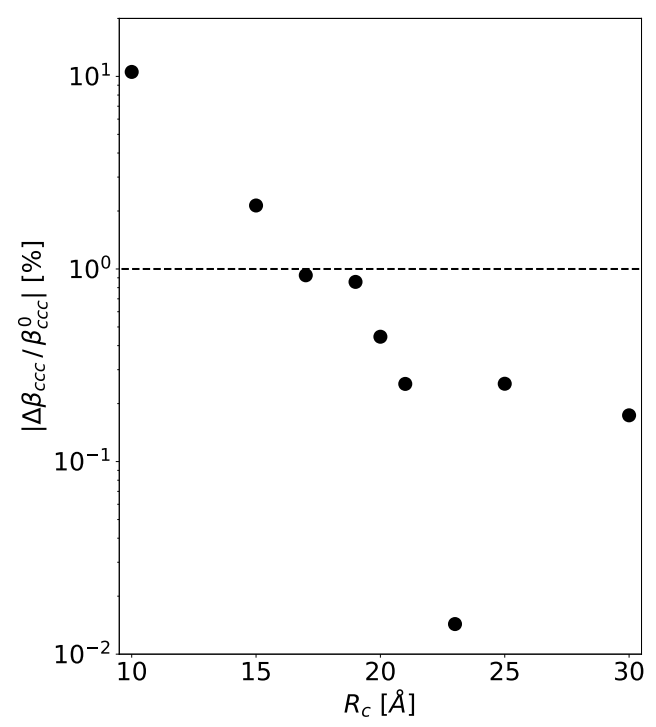

Figure 3: Convergence of the molecular first hyperpolarizability component $\left\langle\beta_{c c c}\right\rangle$ relative to the environment cutoff radius $R_{c}$, for the altitude $-0.06 \pm 0.05 \mathrm{~nm}(\log$. scale). The dashed line represents an error of $1 \%$ [0.1 a.u]. The reference value is obtained for $R_{c}=5 \mathrm{~nm}$. See Figure S10 for other $\beta$ components.

To complete our methodological tests, the convergence of $\left\langle\beta_{c c c}\right\rangle$ relative to the electrostatic environment size $\left(R_{c}\right)$ is illustrated by Figure 3 at the altitude $\Delta Z=-0.06 \mathrm{~nm}$. For the hyperpolarizability, a radius of about $2 \mathrm{~nm}$ is needed to reach a relative error below $1 \%$ [0.1 a.u]. In the following, the neighborhood is therefore built up to a distance $R_{c}=2.3 \mathrm{~nm}$. This behavior is similar to our results for bulk phase (see Figure S9). It highlights that the hyperpolarizability is sensitive to the electrostatic environment up to several nanometers: for aqueous interfaces in general, the S-SHG signal of the BIL might be influ- 
enced indirectly by the structure and composition of a thicker interfacial layer. Therefore, special care should be granted to the choice of the electrostatic embedding description for QM/MM SHG studies. ${ }^{43,59}$

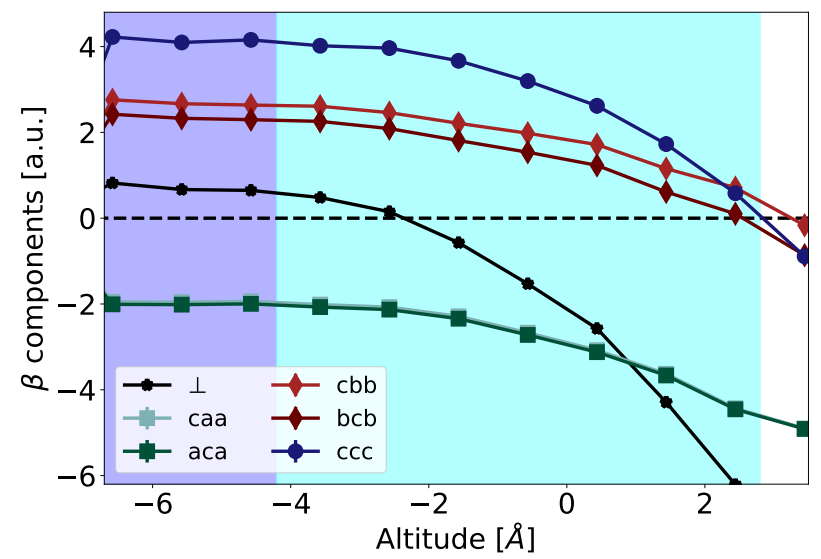

Figure 4: Averages of the hyperpolarizability components $\beta(-2 \omega, \omega, \omega)$ in the molecular frame $\{a, b, c\}$ as a function of the altitude, for an exciting wavelength of $800 \mathrm{~nm}$. $\beta_{\text {caa }}$ and $\beta_{\text {aca }}$ are indistinguishable. $\beta_{\perp}$ is defined by Eq.1. The errors bars, calculated using Eq. S3, are smaller than the symbols.

Finally, we discuss the impact of the interface and report the molecular hyperpolarizability as a function of the altitude in Figure 4. Noticeably, the average hyperpolarizability tensor respects the $\mathrm{C}_{2 v}$ symmetry, both in the bulk (see Table S2) and at the interface. Indeed, the $\mathrm{C}_{2 v}$-forbidden $\beta$ components remain negligible (less than 0.3 a.u. in average) throughout the whole interface, but their fluctuations are large (see Figure S11), as already noted by Liang et al. ${ }^{35}$ for the bulk phase. Concerning the non-vanishing hyperpolarizability components, Figure 4 shows that they start to deviate from their bulk values roughly at the same altitude as the other molecular properties. Concerning the frequency dispersion, characterized for example by the difference between $\left\langle\beta_{c b b}\right\rangle$ and $\left\langle\beta_{b c b}\right\rangle$, it is much smaller in the water bulk phase $(\simeq 0.5$ a.u. $)$ than in the vacuum phase $(\simeq 2.5$ a.u., see Table 1$)$. In the interface region, the frequency dispersion remains weak, and the Kleinman symmetry is practically respected, i.e. $\left\langle\beta_{c a a}\right\rangle=\left\langle\beta_{a c a}\right\rangle$ and $\left\langle\beta_{c b b}\right\rangle \simeq\left\langle\beta_{b c b}\right\rangle$. Therefore, within our 
methodology, the Kleinman symmetry breaking observed experimentally seems difficult to be explained solely by the individual dipolar molecular responses (see Section S4). More precise QM/MD calculations, local effective field effects, collective effects, or quadrupolar contributions should be further explored to explain this behavior.

The evolutions of individual components are relatively modest, and the hyperpolarizability tensor remains closer to the bulk one than to the gaz one. Indeed, similarly to the structural properties depicted in Figure 2, the electric field generated by the point-charge environment differs from the bulk one in the interface area. However, its projection along the molecular c-axis loses only about 30\% of its bulk value (see Figure S5). We attribute this strong remaining field to the presence of close neighbors, and H-bonds, even for the most external layer of water molecules, ${ }^{39}$ see Figure S3.

Even if the variations of individual molecular hyperpolarizability components could be considered as weak, the evolution of $\beta_{c c c}$ and $\beta_{\perp}$ are significant for the S-SHG interpretation. Whereas $\beta_{c c c}$ is dominant in the bulk (4.0 vs 0.5 a.u.), at the altitude of $0.04 \mathrm{~nm}, \beta_{c c c}$ and $\beta_{\perp}$ have opposite values so that $\left(\beta_{c c c}-\beta_{\perp}\right)$ vanishes. The ratio of the two components of Eq. 1 is strongly modulated by the altitude. This questions some frameworks used to analyze S-SHG signals, in which the ratio between $\beta_{c c c}$ and $\beta_{\perp}$ is typically considered as constant throughout the interface (see for example Section S4).

Further, we tackle an even more basic question: can the $\beta$ tensor distribution be replaced by a single, average value at a given altitude? In the dipolar approximation, on may write the susceptibility $\chi^{(2)}$ as proportional to the average over molecular configurations of the product of the rotational matrix $R$ with the $\beta$ tensor. In practice, it is often assumed that all molecules can be described by a single hyperpolarizability tensor $\left\langle\beta_{i j k}\right\rangle$, and that the fluctuations of the hyperpolarizability tensor and the molecular orientation are uncorrelated, i.e. $\left\langle\beta_{i j k} \cos ^{l} \theta\right\rangle=\left\langle\beta_{i j k}\right\rangle\left\langle\cos ^{l} \theta\right\rangle$, for $l=1,2,3$. In our data, such equalities do not hold: Figure 5 illustrates it for Eq. 1, where the contributions of $\beta_{\perp}$ and $\beta_{c c c}$ to $\chi_{Z Z Z}^{(2)}$ are calculated either taking into account the fluctuations, or using the average $\beta$ tensor and average orientation 


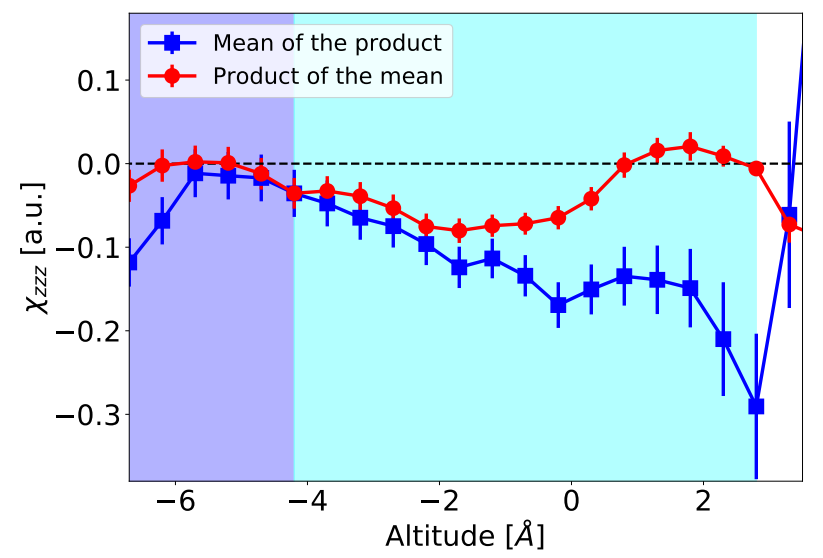

Figure 5: $\quad \chi_{Z Z Z}^{(2)}$ as a function of altitude calculated using Eq. 1, either taking into account the correlations between hyperpolarizability and orientation (blue squares, using $\left\langle\cos ^{l} \theta \beta_{i j k}\right\rangle$ as in Eq. S12), or neglecting them (red circles, using $\left\langle\cos ^{l} \theta\right\rangle\left\langle\beta_{i j k}\right\rangle$ as in Eq. S13).

at each altitude. The differences emphasize correlations between the molecular orientation and molecular hyperpolarizabilities at the interface. Such correlations have to be taken into account in quantitative interpretations of S-SHG intensities. Orientation/hyperpolarizability correlations have been mentioned by Champagne and co-workers in a more complex system, composed of an organic dye embedded in a lipid bilayer, ${ }^{60}$ and we show here that these may appear even at the neat liquid-vapor interface of water.

In conclusion, a $\mathrm{QM} / \mathrm{MD}$ approach was applied at the water liquid-vapor interface, to compute first hyperpolarizabilities of individual water molecules within the very specific environment where the S-SHG signal is generated. Within our approach, the dipolar contribution to the NLO response appears in a molecular layer of about half a nanometer thickness, where both the molecular orientation and the hyperpolarizability significantly differ from the bulk ones. The molecular first hyperpolarizabilities depend on the molecular environment up to radii larger than $2 \mathrm{~nm}$, so that the $\mathrm{S}-\mathrm{SHG}$ is very sensitive to the electrostatic properties of the interface. The hyperpolarizability tensor elements calculated in our PE approach almost respect the Kleinman symmetry, both in the bulk water phase and at the liquid-vapor interface. The variations of the hyperpolarizability within the interface have a impact on the relative weights of its different components. Moreover, we spotlight that the calculated 
molecular hyperpolarizabilities and molecular orientations are correlated: single average values for the molecular hyperpolarizability tensor and orientation are not sufficient to describe the entire distribution of molecular responses. To summarize, the hyperpolarizability cannot be considered as constant for all water molecules at the liquid-vapor interface. Hence, while our non-polarizable dipolar approach still lacks some ingredients to explain completely the experimental SHG intensities, this work shows that numerical approaches are an essential key to rationalize Second Harmonic signals of aqueous interfaces.

\section{Computational Method:}

\section{Molecular Mechanics Simulations}

LAMMPS, ${ }^{51}$ V.11.08.2017 is used to perform the MD simulation along with the TIP4P/2005 water force field. More details are available on the SI. Briefly, 9000 rigid TIP4P/2005 water molecules are placed in a simulation box $\left(5 \times 5 \times 40 \mathrm{~nm}^{3}\right)$ to form a water film, about $10 \mathrm{~nm}$ thick, see Figure 1 (a). The target temperature is $300 \mathrm{~K}$ using a Nose-Hoover thermostat. 3 ns of NVT equilibration is performed before the 20 ns production runs.

To compute the structural properties of the simulated system, such as the molecular orientation and the hyperpolarizabilities, a home-made software FROG is used. This python script opens trajectories from MD codes using the module MDAnalysis ${ }^{61}$ and writes input files for the DALTON software, ${ }^{56}$ release 2018.2 , in order to compute NLO properties within the PE framework.

\section{Response properties within the Density Functional Theory}

The hyperpolarizability depends on electronic degree of freedom, which will be approach using the DFT framework. Then, using frequency-dependent quadratic response scheme, ${ }^{57}$ the hyperpolarizability is extracted. The functional used is CAM-B3LYP ${ }^{62}$ since it compares well with Couple-Cluster Single Double results in the vacuum, see Table S1, and in the bulk phase, see Table 1. The basis set used is large, d-aug-cc-VTZ, and often used for 
water, ${ }^{9,32,35}$ see Figure S2 for the basis convergence in the bulk phase. In order to model the liquid phase effect in our one-molecule based QM calculation, the electrostatic environment is considered. This heterogeneous and discrete solvation model is still in active development. ${ }^{43,50,59,63,64}$ Even if potential improvement can be gained using more accurate electrostatic description of the neighbors ${ }^{59}$ or the calculation of the local effective field, ${ }^{43}$ we have used a point charge description (PE0 scheme in DALTON) of the electrostatic environment fully coherent with the MD simulation protocol. This ensures consistency within the QM embedding and the MD structures and provides a first robust and simple calculation. Regarding the frequency-dependence, the calculations do not take into account the effective field (cavity field) effect, which is beyond the scope of this communication. However, it should not affect much this conclusion: the dispersion of the water polarizability between 800 and $400 \mathrm{~nm}$ is very small. ${ }^{9}$ Our results match well the ones obtained with PE1, ${ }^{32}$ and Liang et al. ${ }^{35}$ have shown the small impact of the MD force field on $\beta$ values for water in bulk phase.

\section{Time and space averaging}

The $\beta(-2 \omega, \omega, \omega)$ time auto-correlation was computed for molecules in the bulk and at the interface (see Fig.S6) ; on the basis of these results, treated MD-frames are time-separated by $20 \mathrm{ps}$, which is sufficient to avoid time-correlation. The center of mass of the whole system is fixed during the MD. To avoid sampling artefacts, the different altitudes composing the interface are not time-separated: each frame treated contributes to all the altitudes. Therefore, in our results we estimated space-correlations, see Section S1.3 for details. Figure S7 displays the convergence of the mean $\beta(-2 \omega, \omega, \omega)$ at $Z-Z_{0}=-0.06 \mathrm{~nm}$ as a function of the number of hyperpolarizability calculations. About 80 time-frames lead to time-converged results; they represent approximately 4000 configurations for this altitude and cost about 1000 hours for 1 standard CPU. Regarding the choice of the altitude $\Delta Z=-0.06 \mathrm{~nm}$ : for this slice, the structural properties differ strongly from the bulk ones while the density remains large 
enough to obtain precise averages. Moreover, due to the large number of molecules with a net orientation, this part is expected to contribute strongly to the second harmonic response of the interface. Therefore, both the time-averaging and $R_{c}$ convergence (see Figure 3) studies have been performed at this altitude. Other altitude were also tested, leading to similar conclusions - data not shown. Finally, our configuration-convergence study focused on the hyperpolarizability components $\beta$. Since the $\chi^{(2)}$ elements also include molecular orientations, their convergence is more demanding. In Figure 5, the $\chi^{(2)}$ is expected to vanish in the bulk region. The small deviations in this area indicate that the convergence relative to sampling is not completely achieved. However, the differences between the two ways of calculating $\chi^{(2)}$ on our data exceed these errors.

\section{Acknowledgement}

We thanks Zacharie Behel for his help during the writing of FROG. We gratefully acknowledge support from the PSMN (Pôle Scientifique de Modélisation Numérique) of the Ecole Normale Supérieure de Lyon for the computing resources.

\section{Supporting Information Available}

The following file Supplementary_Lebreton.pdf are available free of charge and contains:

1. Details of the QM/MD method and comparison with literature.

2. MD structural analysis.

3. Molecular water hyperpolarizability analysis in the bulk and at the liquid/vapor interface (time auto-correlation function and error calculation, time convergence).

4. Susceptibility expression for the study of molecular orientation/ $\beta$ correlations. 


\section{References}

(1) Bian, H.-t.; Feng, R.-r.; Xu, Y.-y.; Guo, Y.; Wang, H.-f. Increased interfacial thickness of the $\mathrm{NaF}, \mathrm{NaCl}$ and $\mathrm{NaBr}$ salt aqueous solutions probed with non-resonant surface second harmonic generation (SHG). Physical Chemistry Chemical Physics 2008, 10, 4920-4931.

(2) Bian, H.-t.; Feng, R.-r.; Guo, Y.; Wang, H.-f. Specific Na+ and K+ cation effects on the interfacial water molecules at the air/aqueous salt solution interfaces probed with nonresonant second harmonic generation. The Journal of Chemical Physics 2009, 130, 134709.

(3) Allen, H. C.; Casillas-Ituarte, N. N.; Sierra-Hernandez, M. R.; Chen, X.; Tang, C. Y. Shedding light on water structure at air-aqueous interfaces: ions, lipids, and hydration. Physical Chemistry Chemical Physics 2009, 11, 5538-5549.

(4) Sulpizi, M.; Salanne, M.; Sprik, M.; Gaigeot, M.-P. Vibrational sum frequency generation spectroscopy of the water liquid--vapor interface from density functional theorybased molecular dynamics simulations. The Journal of Physical Chemistry Letters $\mathbf{2 0 1 2}, \underline{4}, 83-87$.

(5) Chen, Y.; Okur, H. I.; Gomopoulos, N.; Macias-Romero, C.; Cremer, P. S.; Petersen, P. B.; Tocci, G.; Wilkins, D. M.; Liang, C.; Ceriotti, M. et al. Electrolytes induce long-range orientational order and free energy changes in the H-bond network of bulk water. Science Advances 2016, 2, 1-9.

(6) Sengupta, S.; Moberg, D. R.; Paesani, F.; Tyrode, E. Neat water-vapor interface: proton continuum and the nonresonant background. The Journal of Physical Chemistry Letters 2018, 9, 6744-6749.

(7) Ohno, P. E.; Chang, H.; Spencer, A. P.; Liu, Y.; Boamah, M. D.; Wang, H.-f.; 
Geiger, F. M. Beyond the gouy-chapman model with heterodyne-detected second harmonic generation. The Journal of Physical Chemistry Letters 2019, 10, 2328-2334.

(8) Dalstein, L.; Chiang, K.-Y.; Wen, Y.-C. Direct Quantification of Water Surface Charge by Phase-Sensitive Second Harmonic Spectroscopy. The Journal of Physical Chemistry Letters 2019, 10, 5200-5205.

(9) Pham, T. T.; Jonchère, A.; Dufrêche, J. F.; Brevet, P. F.; Diat, O. Analysis of the second harmonic generation signal from a liquid/air and liquid/liquid interface. Journal of Chemical Physics 2017, 146.

(10) Dreier, L. B.; Bernhard, C.; Gonella, G.; Backus, E. H.; Bonn, M. Surface Potential of a Planar Charged Lipid-Water Interface. What Do Vibrating Plate Methods, Second Harmonic and Sum Frequency Measure? The Journal of Physical Chemistry Letters 2018, 9, 5685-5691.

(11) Campen, R. K.; Pymer, A. K.; Nihonyanagi, S.; Borguet, E. Linking surface potential and deprotonation in nanoporous silica: Second harmonic generation and acid/base titration. The Journal of Physical Chemistry C 2010, 114, 18465-18473.

(12) Xu, S.; Xing, S.; Pei, S.-S.; Ivanišť̌̌ev, V.; Lynden-Bell, R.; Baldelli, S. Molecular response of 1-butyl-3-methylimidazolium dicyanamide ionic liquid at the graphene electrode interface investigated by sum frequency generation spectroscopy and molecular dynamics simulations. The Journal of Physical Chemistry C 2015, 119, 26009-26019.

(13) Marchioro, A.; Bischoff, M.; Lütgebaucks, C.; Biriukov, D.; Předota, M.; Roke, S. Surface Characterization of Colloidal Silica Nanoparticles by Second Harmonic Scattering: Quantifying the Surface Potential and Interfacial Water Order. The Journal of Physical Chemistry C 2019, 123, 20393-20404.

(14) Raberg, J. H.; Vatamanu, J.; Harris, S. J.; van Oversteeg, C. H.; Ramos, A.; Borodin, O.; Cuk, T. Probing electric double-layer composition via in situ vibrational 
spectroscopy and molecular simulations. The Journal of Physical Chemistry Letters 2019, 10, 3381-3389.

(15) Pezzotti, S.; Galimberti, D. R.; Gaigeot, M.-P. Deconvolution of BIL-SFG and DLSFG spectroscopic signals reveals order/disorder of water at the elusive aqueous silica interface. Physical Chemistry Chemical Physics 2019, 21, 22188-22202.

(16) Shen, Y. Optical Second Harmonic Generation At Interfaces. Annual Review of Physical Chemistry 1989, 40, 327-350.

(17) Wei, Q.; Zhou, D.; Bian, H. Molecular structure and adsorption of dimethyl sulfoxide at the air/aqueous solution interface probed by non-resonant second harmonic generation. Phys. Chem. Chem. Phys. 2018, 20, 11758-11767.

(18) Donohue, E.; Khorsand, S.; Mercado, G.; Varney, K. M.; Wilder, P. T.; Yu, W.; MacKerell, A. D.; Alexander, P.; Van, Q. N.; Moree, B. et al. Second harmonic generation detection of Ras conformational changes and discovery of a small molecule binder. Proceedings of the National Academy of Sciences of the United States of America 2019, 116, 17290-17297.

(19) Ong, S.; Zhao, X.; Eisenthal, K. B. Polarization of water molecules at a charged interface: second harmonic studies of the silica/water interface. Chemical Physics Letters 1992, 191, 327-335.

(20) Liu, Y.; Yan, E. C.; Zhao, X.; Eisenthal, K. B. Surface potential of charged liposomes determined by second harmonic generation. Langmuir 2001, 17, 2063-2066.

(21) de Beer, A. G. F.; Roke, S. Obtaining molecular orientation from second harmonic and sum frequency scattering experiments in water: Angular distribution and polarization dependence. The Journal of Chemical Physics 2010, 132, 234702. 
(22) Tocci, G.; Liang, C.; Wilkins, D. M.; Roke, S.; Ceriotti, M. Second-Harmonic Scattering as a Probe of Structural Correlations in Liquids. Journal of Physical Chemistry Letters 2016, $\underline{7}, 4311-4316$.

(23) Pezzotti, S.; Galimberti, D. R.; Shen, Y. R.; Gaigeot, M.-P. Structural definition of the BIL and DL: A new universal methodology to rationalize non-linear $\chi^{(2)}(\omega)$ SFG signals at charged interfaces, including $\chi^{(3)}(\omega)$ contributions. Physical Chemistry Chemical Physics 2018, 20, 5190-5199.

(24) Bouquiaux, C.; Tonnelé, C.; Castet, F.; Champagne, B. Second-Order Nonlinear Optical Properties of an Amphiphilic Dye Embedded in a Lipid Bilayer. A Combined Molecular Dynamics-Quantum Chemistry Study. The Journal of Physical Chemistry B 2020, 124, 2101-2109.

(25) Roke, S. Nonlinear Optical Spectroscopy of Soft Matter Interfaces. ChemPhysChem 2009, 10, 1380-1388.

(26) Nasir, M. N.; Benichou, E.; Loison, C.; Russier-Antoine, I.; Besson, F.; Brevet, P.-F. Influence of the tyrosine environment on the second harmonic generation of iturinic antimicrobial lipopeptides at the air-water interface. Physical Chemistry Chemical Physics 2013, 15, 19919-19924.

(27) Loison, C.; Nasir, M. N.; Benichou, E.; Besson, F.; Brevet, P.-F. Multi-scale modeling of mycosubtilin lipopeptides at the air/water interface: structure and optical second harmonic generation. Physical Chemistry Chemical Physics 2014, 16, 2136-2148.

(28) Hartkamp, R.; Biance, A.-L.; Fu, L.; Dufrêche, J.-F.; Bonhomme, O.; Joly, L. Measuring surface charge: Why experimental characterization and molecular modeling should be coupled. Current Opinion in Colloid \& Interface Science 2018, 37, 101-114.

(29) Zhang, W.-k.; Zheng, D.-s.; Xu, Y.-y.; Bian, H.-t.; Guo, Y.; Wang, H.-f. Reconsideration of second-harmonic generation from isotropic liquid interface: Broken Kleinman sym- 
metry of neat air/water interface from dipolar contribution. The Journal of Chemical Physics 2005, 123, 224713.

(30) Kaatz, P.; Donley, E. A.; Shelton, D. P. A comparison of molecular hyperpolarizabilities from gas and liquid phase measurements. The Journal of Chemical Physics 1998, 108, 849-856.

(31) Poulsen, T. D.; Ogilby, P. R.; Mikkelsen, K. V. A quantum mechanical method for calculating nonlinear optical properties of condensed phase molecules coupled to a molecular mechanics field: A quadratic multiconfigurational self-consistent-field/molecular mechanics response method. The Journal of Chemical Physics 2001, 115, 7843-7851.

(32) Osted, A.; Kongsted, J.; Mikkelsen, K. V.; Åstrand, P. O.; Christiansen, O. Statistical mechanically averaged molecular properties of liquid water calculated using the combined coupled cluster/molecular dynamics method. Journal of Chemical Physics 2006, 124.

(33) Nielsen, C. B.; Christiansen, O.; Mikkelsen, K. V.; Kongsted, J. Density functional self-consistent quantum mechanics/molecular mechanics theory for linear and nonlinear molecular properties: Applications to solvated water and formaldehyde. The Journal of Chemical Physics 2007, 126, 154112.

(34) Harczuk, I.; Vahtras, O.; Ågren, H. Hyperpolarizabilities of extended molecular mechanical systems. Physical Chemistry Chemical Physics 2016, 18, 8710-8722.

(35) Liang, C.; Tocci, G.; Wilkins, D. M.; Grisafi, A.; Roke, S.; Ceriotti, M. Solvent fluctuations and nuclear quantum effects modulate the molecular hyperpolarizability of water. Physical Review B 2017, 96, 1-6.

(36) Guyot-Sionnest, P.; Shen, Y. Bulk contribution in surface second-harmonic generation.

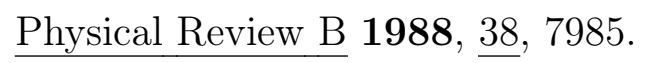


(37) Dailey, C. A.; Burke, B. J.; Simpson, G. J. The general failure of Kleinman symmetry in practical nonlinear optical applications. Chemical Physics Letters 2004, 390, 8-13.

(38) Kongsted, J.; Osted, A.; Mikkelsen, K. V.; Christiansen, O. Second harmonic generation second hyperpolarizability of water calculated using the combined coupled cluster dielectric continuum or different molecular mechanics methods. Journal of Chemical Physics 2004, 120, 3787-3798.

(39) Pezzotti, S.; Galimberti, D. R.; Gaigeot, M. P. 2D H-Bond Network as the Topmost Skin to the Air-Water Interface. Journal of Physical Chemistry Letters 2017, $\underline{8}, 3133-3141$.

(40) Mikkelsen, K. V.; Luo, Y.; Ågren, H.; Jørgensen, P. Sign change of hyperpolarizabilities of solvated water. The Journal of Chemical Physics 1995, 102, 9362-9367.

(41) Garrett, K.; Sosa Vazquez, X.; Egri, S. B.; Wilmer, J.; Johnson, L. E.; Robinson, B. H.; Isborn, C. M. Optimum exchange for calculation of excitation energies and hyperpolarizabilities of organic electro-optic chromophores. Journal of Chemical Theory and Computation 2014, 10, 3821-3831.

(42) Osella, S.; Murugan, N. A.; Jena, N. K.; Knippenberg, S. Investigation into biological environments through (non) linear optics: A multiscale study of laurdan derivatives. Journal of Chemical Theory and Computation 2016, 12, 6169-6181.

(43) List, N. H.; Jensen, H. J. A.; Kongsted, J. Local electric fields and molecular properties in heterogeneous environments through polarizable embedding. Physical Chemistry Chemical Physics 2016, 18, 10070-10080.

(44) Licari, G.; Cwiklik, L.; Jungwirth, P.; Vauthey, E. Exploring Fluorescent Dyes at Biomimetic Interfaces with Second Harmonic Generation and Molecular Dynamics. Langmuir 2017, 33, 3373-3383. 
(45) De Wergifosse, M.; Botek, E.; De Meulenaere, E.; Clays, K.; Champagne, B. ONIOM Investigation of the second-order nonlinear optical responses of fluorescent proteins. The Journal of Physical Chemistry B 2018, 122, 4993-5005.

(46) Tonnelé, C.; Champagne, B.; Muccioli, L.; Castet, F. Nonlinear Optical Contrast in Azobenzene-Based Self-Assembled Monolayers. Chemistry of Materials 2019, 31, 67596769.

(47) Cardenuto, M. H.; Champagne, B. The first hyperpolarizability of nitrobenzene in benzene solutions: investigation of the effects of electron correlation within the sequential QM/MM approach. Physical Chemistry Chemical Physics 2015, 17, 23634-23642.

(48) de Wergifosse, M.; Castet, F.; Champagne, B. Frequency dispersion of the first hyperpolarizabilities of reference molecules for nonlinear optics. The Journal of Chemical Physics 2015, 142, 194102.

(49) Beaujean, P.; Champagne, B. Coupled cluster evaluation of the second and third harmonic scattering responses of small molecules. Theoretical Chemistry Accounts 2018, $\underline{137}, 50$.

(50) Steinmann, C.; Reinholdt, P.; Nørby, M. S.; Kongsted, J.; Olsen, J. M. H. Response properties of embedded molecules through the polarizable embedding model. International Journal of Quantum Chemistry 2019, 119, e25717.

(51) Plimpton, S. Fast Parallel Algorithms for Short âĂŞ Range Molecular Dynamics. Journal of Computational Physics 1995, 117, 1-19.

(52) Bu, W.; Kim, D.; Vaknin, D. Density Profiles of Liquid/Vapor Interfaces Away from Their Critical Points. The Journal of Physical Chemistry C 2014, 118, 12405-12409.

(53) Brevet, P.-F. Surface Second Harmonic Generation; PPUR presses polytechniques, 1997. 
(54) Gan, W.; Wu, D.; Zhang, Z.; Feng, R. R.; Wang, H. F. Polarization and experimental configuration analyses of sum frequency generation vibrational spectra, structure, and orientational motion of the air/water interface. Journal of Chemical Physics 2006, 124.

(55) Olsen, J. M.; Aidas, K.; Kongsted, J. Excited states in solution through polarizable embedding. Journal of Chemical Theory and Computation 2010, $\underline{6}$, 3721-3734.

(56) Aidas, K.; Angeli, C.; Bak, K. L.; Bakken, V.; Bast, R.; Boman, L.; Christiansen, O.; Cimiraglia, R.; Coriani, S.; Dahle, P. et al. The Dalton quantum chemistry program system. Wiley Interdisciplinary Reviews: Computational Molecular Science 2014, $\underline{4}$, 269-284.

(57) Sałek, P.; Vahtras, O.; Helgaker, T.; Ågren, H. Density-functional theory of linear and nonlinear time-dependent molecular properties. The Journal of Chemical Physics 2002, 117, 9630-9645.

(58) Kongsted, J.; Osted, A.; Mikkelsen, K. V.; Christiansen, O. Nonlinear optical response properties of molecules in condensed phases using the coupled cluster/dielectric continuum or molecular mechanics methods. Journal of Chemical Physics 2003, 119, 10519 10535.

(59) Beerepoot, M. T.; Steindal, A. H.; List, N. H.; Kongsted, J.; Olsen, J. M. H. Averaged Solvent Embedding Potential Parameters for Multiscale Modeling of Molecular Properties. Journal of Chemical Theory and Computation 2016, 12, 1684-1695.

(60) Bouquiaux, C.; TonnelÃl', C.; Castet, F.; Champagne, B. Second-Order Nonlinear Optical Properties of an Amphiphilic Dye Embedded in a Lipid Bilayer. A Combined Molecular Dynamics-Quantum Chemistry Study. The Journal of Physical Chemistry B 2020, 124, 2101-2109.

(61) Michaud-Agrawal, N.; Denning, E. J.; Woolf, T. B.; Beckstein, O. MDAnalysis: a 
toolkit for the analysis of molecular dynamics simulations. Journal of computational chemistry 2011, 32, 2319-2327.

(62) Yanai, T.; Tew, D. P.; Handy, N. C. A new hybrid exchangeâĂŞcorrelation functional using the Coulomb-attenuating method (CAM-B3LYP). 2008, 393, 51-57.

(63) Kjellgren, E. R.; Haugaard Olsen, J. M.; Kongsted, J. Importance of Accurate Structures for Quantum Chemistry Embedding Methods: Which Strategy Is Better? Journal of Chemical Theory and Computation 2018, 14, 4309-4319.

(64) Marefat Khah, A.; Reinholdt, P.; Olsen, J. M. H.; Kongsted, J.; Hattig, C. Avoiding Electron Spill-Out in QM/MM Calculations on Excited States with Simple Pseudopotentials. Journal of Chemical Theory and Computation 2020, 16, 1373-1381. 